\title{
The Role of Reactant Transport in Determining the Properties of NIF Shells Made by Interfacial Polycondensation
}

\author{
K.E. Hamilton \\ S.A. Letts \\ S.R. Buckley \\ E.M. Fearon \\ G. Wilemski \\ R.C. Cook \\ D.Schroen-Carey \\ W.J. Schafer and Associates, Livermore, California \\ This paper was prepared for submittal to the \\ Eleventh Target Fabrication Specialists' Meeting \\ Orcas Island, Washington \\ September 8-12, 1996
}

March 26, 1997

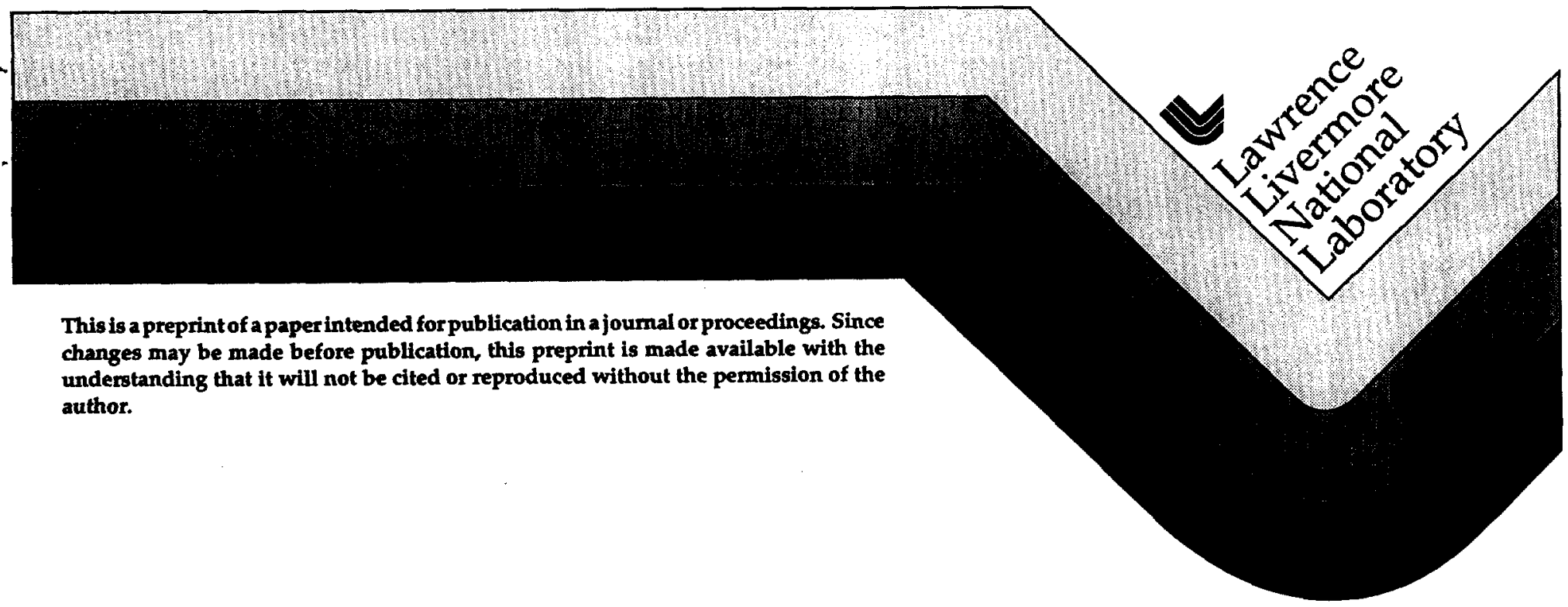




\section{DISCLAIMER}

This document was prepared as an account of work sponsored by an agency of the United States Government. Neither the United States Government nor the University of California nor any of their employees, makes any warranty, express or implied, or assumes any legal liability or responsibility for the accuracy, completeness, or usefulness of any information, apparatus, product, or process disclosed, or represents that its use would not infringe privately owned rights. Reference herein to any specific commercial product, process, or service by trade name, trademark, manufacturer, or otherwise, does not necessarily constitute or imply its endorsement, recommendation, or favoring by the United States Government or the University of Califomia. The views and opinions of authors expressed herein do not necessarily state or reflect those of the United States Government or the University of California, and shall not be used for advertising or product endorsement purposes. 


\section{THE ROLE OF REACTANT TRANSPORT IN DETERMINING THE PROPERTIES OF NIF SHELLS MADE BY INTERFACIAL POLYCONDENSATION}

\author{
Kenneth E. Hamilton, Stephan A. Letts, \\ Steven R. Buckley, Evelyn M. Fearon, Gerald Wilemski, \\ and Robert C. Cook \\ Lawrence Livermore National Laboratory \\ P.O. Box 808, L-474, Livermore CA 94551
} (510) 422-0936

\author{
Diana Schroen-Carey \\ W. J. Schafer and Associates \\ 303 Lindbergh Ave., Livermore CA 94550 \\ (510) 447-0555
}

\section{ABSTRACT}

Polymer shells up to $2 \mathrm{~mm}$ in diameter were prepared using an interfacial polycondensation / cross-linking reaction occurring at the surface of an oil drop. The oil phase is comprised of a solution (20 wt\% or less) of isophthaloyl dichloride (IPC) dissolved in an organic solvent. An interfacial reaction is initiated when the IPCloaded oil drop is submerged in an aqueous solution of poly(p-vinylphenol) (PVP), a poly(electrolyte) at elevated $\mathrm{pH}$. Composition, structure, and surface finish for fullyformed dry shells were assessed using a number of techniques including scanning electron microscopy (SEM), atomic force microscopy (AFM), fourier-transform infrared spectroscopy (FTIR), pyrolysis-gas chromatography (GC) mass spectroscopy (MS), microhardness measurements, gas permeability, and solvent permeability measurements. From deposition rate data, a reaction mechanism and key reaction parameters were identified.

The deposition rate of shell membrane material was found to be a diffusion limited reaction of IPC through the forming membrane to the exterior shell interface (which is believed to be the reaction front). The final thickness of the film deposited at the interface and the rate of deposition were found to be strong functions of the IPC concentration and oil phase solvent. Films made with diethyl phthalate (DEP) were thinner and harder than films made using 1,6dichlorohexane (DCH) as a solvent. Differences in solubility of the forming membrane in DCH and DEP appear to be able to account for the differences in deposition rate and the hardness (related to cross-linking density). The deposition can be thought of as a phase separation which is affected by both the poly(electrolyte)/ ionomer transition and the amount of cross-linking. Finally, it was found that the choice of oil phase solvent profoundly affects the evolution of the outer surface roughness.

\section{INTRODUCTION}

In several years the National Ignition Facility (NIF) will require targets that are $2 \mathrm{~mm}$ in diameter with structural specifications on surface finish and uniformity exceeding currently produced capsules. ${ }^{1}$ The current technology utilizing a heated drop tower to blow $0.5 \mathrm{~mm}$ shells from polymer solution does not scale to the larger NIF requirement. ${ }^{2}$ We are exploring several methods for producing large capsules including: a depolymerizing mandrel technique, ${ }^{3.5}$ microencapsulation, ${ }^{6-8}$ ballistic furnace blowing technologies, ${ }^{2-10}$ and the technique described here of interfacial polycondensation.

The interfacial polycondensation method was first investigated for inertial confinement fusion (ICF) capsules as a method for producing a vapor barrier skin $(-2 \mathrm{~mm}$ in thickness) on foam shells. "In this project we explored the possibility of using interfacial polycondensation to produce NIF-scale shells. We envisioned interfacial polycondensation as a method that could possibly produce a wall thickness necessary for NIF shells $(-200 \mu \mathrm{m})$ in a single step, that would avoid vacuole and non-uniformity problems encountered in microencapsulation, and would not be limited to producing thin shells as is the case with drop tower and ballistic furnace methods. By working in a neutrally buoyant solution and having a radially symmetric reaction front, we hoped to be able to produce perfectly spherical shells.

We focused on understanding the shell growth mechanism from which we hoped to better assess the potential for increasing and controlling wall thickness and ultimately improving surface finish. Our approach was to measure the rate of wall growth as a function of process conditions such as starting reactant concentrations (IPC and PVP), oil-phase solvent (primarily DEP and DCH), and reaction time. The dried shells were characterized for structure by measuring permeability and by observing the surface and fracture 
morphologies using SEM and AFM. Composition and information on the cross-linking of the shell was measured by FTIR, pyrolysis-GC-MS, nanoindentation, and permeation.

\section{EXPERIMENTAL}

The interfacial polycondensation method for making large shells involved the reaction of poly(vinyl phenol), PVP (also known as poly(4-hydroxystyrene)), and isophthaloyl dichloride, IPC (Figure 1). The shell generation process entailed five steps: (1) solution preparation; (2) formation of organic droplets; (3) droplet delivery to the aqueous solution where shell wall growth proceeds; (4) solvent rinses and exchanges; and (5) supercritical carbon dioxide exchange / drying. Three solutions were prepared for the interfacial polycondensation reaction: the organic phase containing a diacid chloride dissolved in organic solvent, a stripping phase used in the droplet generator, and the aqueous phase containing PVP (Scientific Polymer Products, $\mathrm{M}_{\mathrm{W}}=-30 \mathrm{~K}$ ). A range of IPC concentrations (2 to $20 \mathrm{wt} \%$ ) were used in the course of this study, and a number of organic solvents were employed including DEP, DCH, 4-chlorotoluene, and chlorocyclohexane. The solvent choice was based on density matching properties and on solvent quality, as well as the effect of their selection on shell wall growth, surface finish, and wall properties. In this report we will concentrate on the results obtained using DEP and DCH which represent extremes in the shell growth behavior.

The stripping solution used for the organic droplet formation was an aqueous solution containing 2 wt\% hydroxyethyl cellulose (HEC, Scientific Polymer Products, $\mathrm{M}_{\mathrm{W}}=-30 \mathrm{~K}$ ). Enough $\mathrm{NaOH}$ was added to maintain a solution $\mathrm{pH}$ close to 12.6. Because the shells had a tendency to aggregate, it also was necessary to add HEC and a small amount of Tween 80 (i.e., 0.6 wt\% sorbitan monooleate) the PVP solution. All solutions were filtered through $0.2 \mu \mathrm{m}$ filters before use.

Droplets of the oil phase were made using a coaxial orifice droplet generator. The oil phase was pumped from a $10 \mathrm{ml}$ glass syringe through the inner orifice of the generator, a $400 \mu \mathrm{m}$ ID stainless steel tube. To control the size of the forming drops, the HEC stripping solution was pumped from a $60 \mathrm{ml}$ syringe through the outer orifice with a $4 \mathrm{~mm}$ inside diameter; this flow stripped the oil droplets off the orifice. Typical flow rates used were $0.2 \mathrm{ml} / \mathrm{min}$. for the inner oil phase and $4 \mathrm{ml} / \mathrm{min}$. for the stripping phase. The oil drops were then carried in the stripping solution through a glass tube ( $4 \mathrm{~mm}$ ID) into a $150 \mathrm{ml}$ vessel $(7.5 \mathrm{~cm}$ diameter $\times 4.0 \mathrm{~cm}$ high) containing the PVP solution. The total volume added by the oil drops can be considered negligible in comparison to the volume of PVP solution. Volumetric flow rates, diameter of the oil orifice, interfacial surface tension, and density differences of the organic and stripping solutions were the key parameters which control droplet size.

To avoid having the droplets settle to the bottom of the collection vessel and to allow the reactants sufficient access to all surfaces of the oil droplets, constant agitation was used. The style of agitation had a significant impact on shell quality, and it is desirable to use a minimum amount of agitation ( $50 \mathrm{RPM}$ with $1 / 8$ inch pitchless impeller

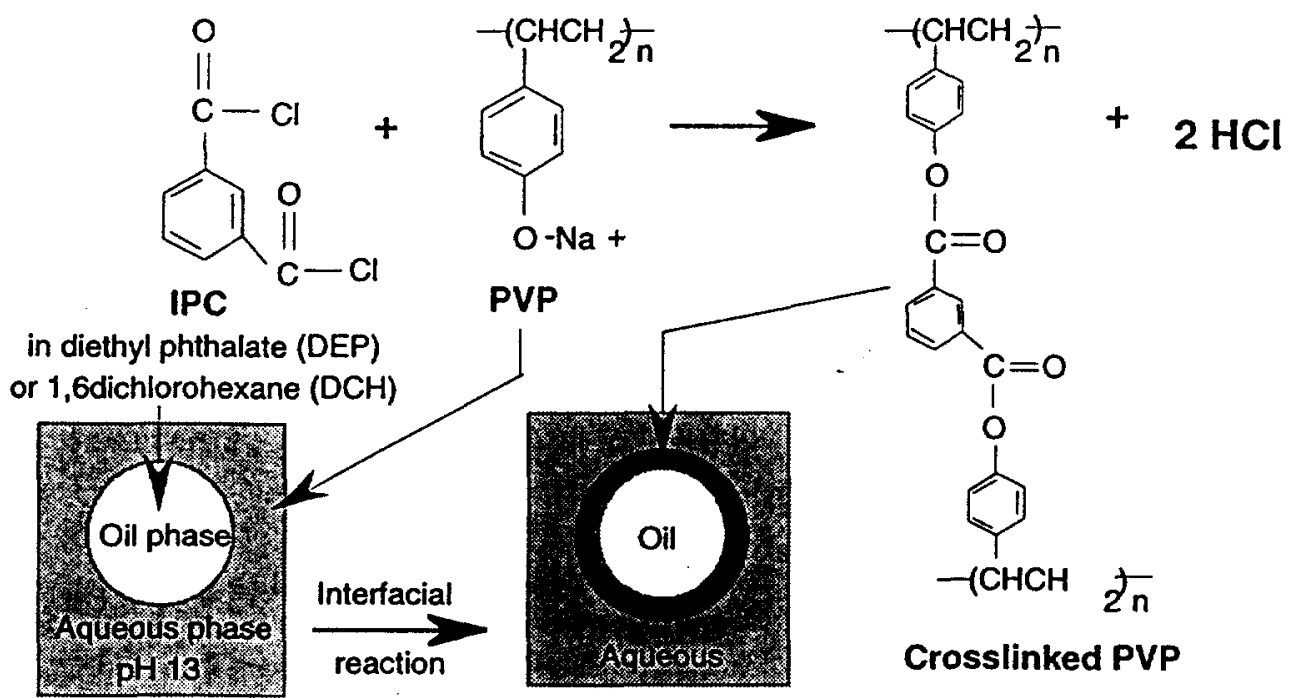

Fig. 1: Reaction scheme used for shell generation with interfacial polycondensation. A droplet of oil phase (composed of IPC in either DEP or DCH) reacts with the surrounding aqueous phase (containing deprotonated PVP chains). 
blades mounted on a 2 inch diameter shaft) so as to sufficiently maintain a suspension without inducing various deformations. Once the desired reaction time had elapsed, the agitation was stopped, and the entrained PVP solution was immediately removed by rinsing with distilled water and a 2:1 ethanol / acetone mixture. To ensure complete removal of the IPC and the solvent, a number of additional, extended, ethanol / acetone exchanges were spaced over two days.

The final step in processing the shells was the exchange of the ethanol / acetone solution for liquid $\mathrm{CO}_{2}$. To accomplish this, the shells were immersed in liquid $\mathrm{CO}_{2}$ in a supercritical extractor. The $\mathrm{CO}_{2}$ was exchanged three or four times daily for a period of four days. Finally, the $\mathrm{CO}_{2}$ was removed from the shells by raising the temperature of the pressure vessel to $45^{\circ} \mathrm{C}$ (above the critical temperature, $32{ }^{\circ} \mathrm{C}$, and critical pressure, $72.8 \mathrm{~atm}$, for $\mathrm{CO}_{2}$ ) at which point the gaseous $\mathrm{CO}_{2}$ was slowly vented leaving dry shells.

\section{RESULTS}

\section{A. Shell Growth}

Initial experiments in this area, covering only a limited range of solution concentrations, produced shells of sufficient sphericity with wall thicknesses up to a maximum of $30 \mu \mathrm{m}$ and with an unacceptably rough surface finish ( $>20 \mathrm{~nm}$ root mean square, RMS, roughness from a $40 \mu \mathrm{m}$ $x 40 \mu \mathrm{m}$ AFM patch scan). We expanded our investigation to include a wider range of IPC concentrations as well as examining different organic solvents from the ones used by Takagi, et al.," for coatings on low density foam mandrels. Figure 2 compares the wall thickness for shells produced using $10 \mathrm{wt} \%$ IPC in two different solvents: DEP and DCH. In DEP wall thickness grew to an upper limit of

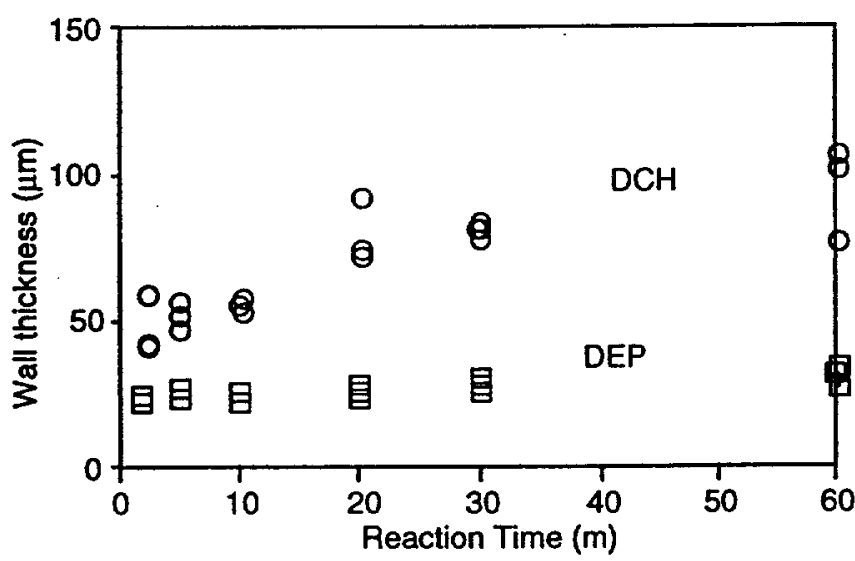

Fig. 2: Wall thickness as a function of reaction time. Both membrane deposition rate and maximum wall thickness are larger for the DCH solvent than the DEP solvent at a constant IPC concentration (10 wt\% IPC in this case). about $30 \mu \mathrm{m}$, while in DCH the thickness grew to about $90 \mu \mathrm{m}$. This discovery showed that the $30 \mu \mathrm{m}$ barrier could be surpassed simply by using an alternate reaction medium. Shells were generated using a range of concentrations from which we found that the shell deposition rate and ultimate wall thickness were a function of IPC concentration (Figure 3). We found that the shell was limited in its growth by the amount of IPC contained inside the shell and by organic solvent choice, although it is not known if there was complete IPC consumption during the reaction.

\section{B. Uniformity}

Shell uniformity was assessed using interferometry. For a fairly uniform shell (both spherical and concentric) one obtains concentric fringe patterns simultaneously in three orthogonal directions. The current processing technique tended to produce less than $1 \%$ of the shells having this level of uniformity. However, solely based on this relatively low yield, interfacial polycondensation should not be eliminated as a viable shell fabrication technique since the other competing technologies have similarly low (or worse) yield.

There are several sources of shell non-uniformity. We found that it was necessary to stir somewhat vigorously to prevent the shells from settling on and adhering to the bottom of the reaction vessel or from aggregating. Temporary adhesion results in contact spots on the shells. Aside

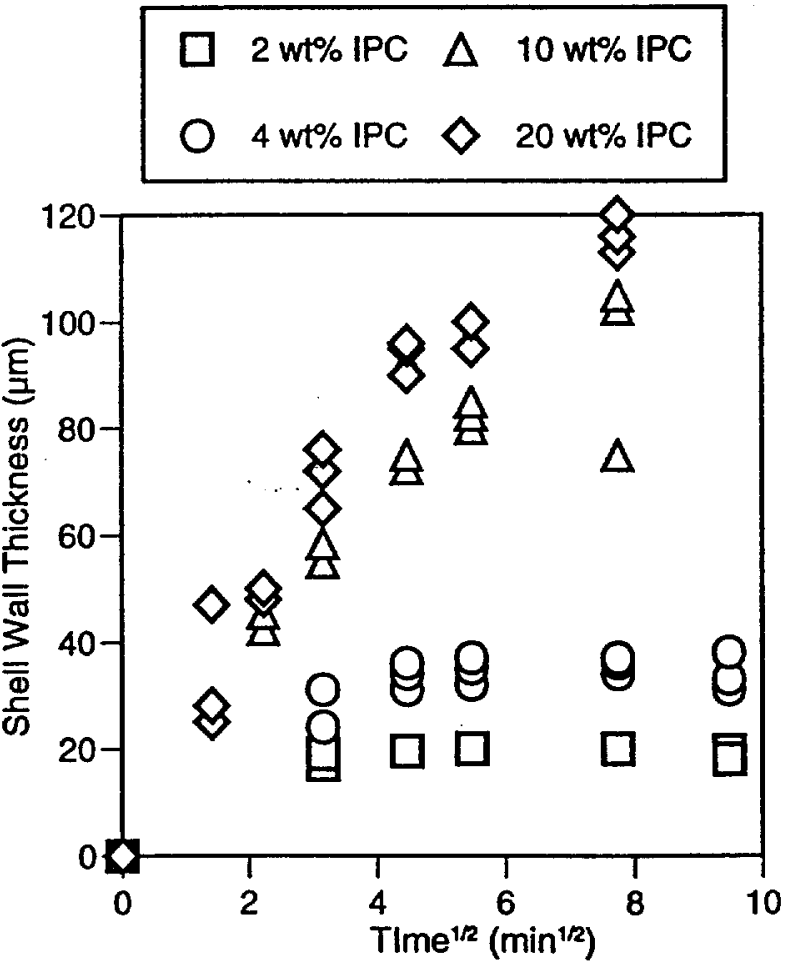

Fig. 3: Shell growth as a function of time for various IPC wt\% in DCH solvent. 
from contact imperfections in the shells, shear induced deformations from the vigorous stirring may be a factor that causes distortions in the shell sphericity. Another possible explanation for the deformation / non-sphericity of the shells is that it is caused, in part, by the volume decrease of the core fluid due to the efflux of IPC to the surface where the reaction with PVP occurs.

\section{Surface Roughness Analysis}

Dried shells were characterized for surface structure primarily using scanning electron microscopy (SEM). The right-hand side of Figure 4 shows a typical surface which appears to be uniformly textured / bumpy. We further. investigated shell structure by fracturing and viewing the cross section. In general, we found the inner surface, shown in Figure 4, to be very smooth in comparison to the outer surface ( $2 \mathrm{~nm}$ RMS vs. $15-50 \mathrm{~nm}$ RMS). Occasionally we observed crystals on the inner surface when the exchange process was incomplete (presumably of hydrolyzed IPC and / or $\mathrm{NaCl}$ ), but for the most part, the inner surface smoothness was quite good. Further investigation of the fracture surface shows that the depth of the inner smooth region is a film only about $100 \mathrm{~nm}$ thick. This structure suggests that the shell initially forms a thin film by a homogeneous reaction mechanism ${ }^{12}$ which is analogous to the nylon rope trick involving an interfacial polymerization from two highly mobile monomers. ${ }^{13}$ Beyond this smooth skin the shell morphology is uniformly granular, and it is maintained all the way to the outer surface without any obvious change in granule size. The change in morphology apparently is due to a change in reaction mechanism.

The bulk of the shell deposition proceeds according to what will be shown later to be a reaction limited by diffusion of the IPC through the growing film to the outer surface where it is able to react with the PVP. We believe the reaction between IPC and deprotonated PVP polyelectrolyte continues in solution up to a critical level at which point the reacted cross-linked polymer becomes insoluble in the interfacial region and phase separates to form clusters of tiny interconnected spheres $(\sim 50 \mathrm{~nm}$ in diameter). The size of the spheres is large in comparison with the radius of gyration for the $30 \mathrm{~K} \mathrm{M}_{\mathrm{W}}$ of PVP, $-8 \mathrm{~nm}$. The extent of reaction (in terms of the percent phenol reacted on the PVP chain) reached before precipitation is dependent on the reaction conditions including organic phase solvent, temperature, and the evolving local solution $\mathrm{pH}$. Further confirmation of this mechanism will be presented in the section on IR composition measurement of the shells.

Fig. 4: At very short reaction times, a very thick skin $(-100 \mathrm{~nm})$ is formed at the inner shell surface as viewed by the crosssectional fracture surface (a). All subsequent shell deposition has a granular nature to it. Shell walls are fairly uniform as viewed from the exterior surface (b). The shell shown here was made in DEP with 4 wt\% IPC. 
(a)

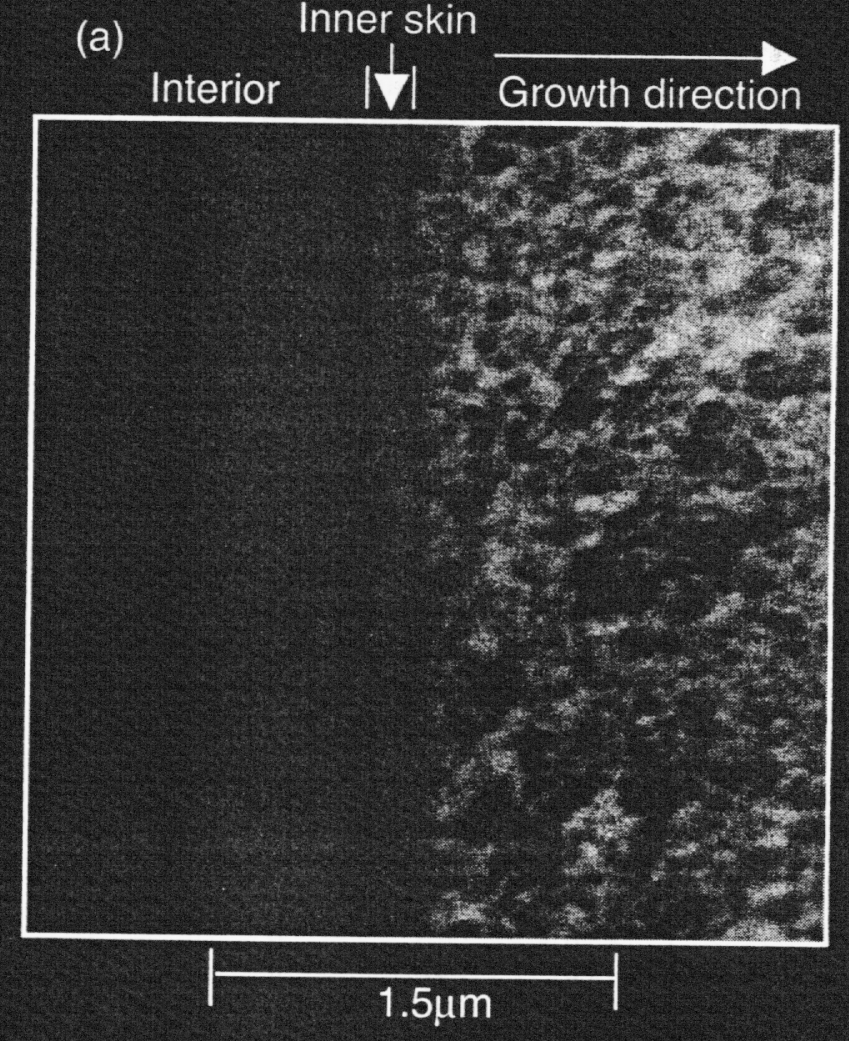

(b)

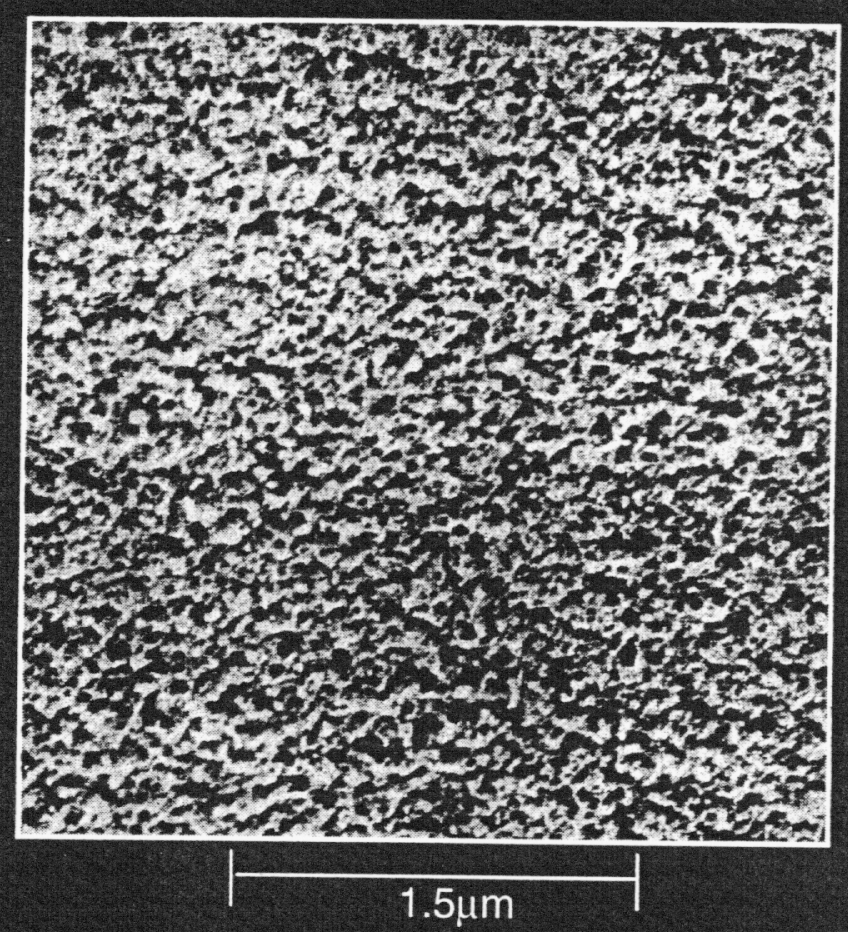


The surface roughness of the dried shells also was evaluated using AFM to scan a representative $40 \mu \mathrm{m} \mathrm{x}$ $40 \mu \mathrm{m}$ patch of the exterior shell surface. By measuring surface roughness as a function of reaction parameters, we hoped to be able to optimize the processing to improve the surface smoothness. Shells made in DEP start with a surface roughness of approximately $40 \mathrm{~nm}$ RMS, and they seem to become smoother with reaction time (Figure 5). This behavior is certainly unusual since it is not clear how it is possible, without the existence of a "planarization" mechanism, for the deposition to be smoother than a previous layer, i.e., the "substrate." In contrast, the surface roughness of the polymer shells made in DCH show increasing roughness with thickness. These shells grow more rapidly possibly because the IPC is able to diffuse through the growing polymer shell faster. The shells made in DCH may also be less cross-linked, and as a result they are more susceptible to swelling during solvent exchanges and the supercritical drying process. The comments about cross-linking are consistent with analysis of shell composition by FTIR which will be shown later.

To produce a smooth outer surface, we inverted the phases by dispersing aqueous PVP droplets in an organic outer phase containing the IPC. We thought the impact of the IPC hydrolysis side-reaction could be minimized by increasing the amount of IPC available. Shells made using inverted phases have outer surface roughness of $2 \mathrm{~nm} \mathrm{RMS}$; however, the inner surface has the same textured finish we previously observed on the outside. Clearly, we did not alter the physical processes which control the development of surface structure by merely reversing the phases, but we have identified where the initial reaction front occurs and the direction of propagation.

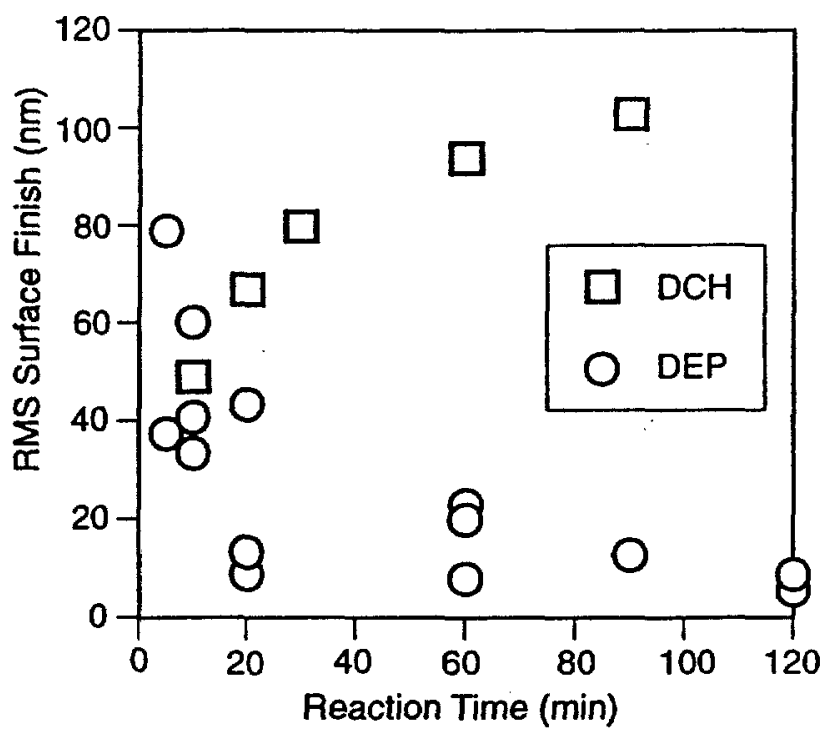

Fig. 5: Evolution of surface finish for the interfacial polycondensation shells in DEP and DCH solvents at 4 wt\% IPC.

\section{IR Composition Analysis}

IR was used to characterize interfacial polycondensation shells to determine the effect of reaction conditions on composition. Figure 6 compares the IR spectrum of the two starting materials, PVP and IPC, to the copolymer formed at the interface. Several absorbance bands potentially could be used to make structure and composition measurements on the shell. The carbonyl stretching vibration at $1740 \mathrm{~cm}^{-1}$ is particularly attractive due to the absence of interferences in the PVP spectrum. The carbonyl absorption in IPC occurs as a doublet at $1720 \mathrm{~cm}^{-1}$ and $1700 \mathrm{~cm}^{-1}$; however, these bands could not be resolved. As apparent from Figure 1, the IPC reacts with PVP by forming ester linkages which incorporates carbonyl groups in the copolymer film. From measurements of the magnitude of the carbonyl absorption, we were able to determine the relative IPC concentration in the polymer shell.

The shells were weighed and mixed with desiccated $\mathrm{KBr}$ powder. The mixture was ground in a mortar and pestle, pressed into a pellet and analyzed by FTIR. The carbonyl absorbance peaks were corrected for background and integrated. We then calculated the normalized absorbance by dividing by the initial mass of the shell. This normalized absorption is proportional to the IPC concentration in the shell. We measured the carbonyl absorption per $\mu \mathrm{g}$ (related to twice the IPC concentration) as a function of reaction time for shells made in DEP and DCH; we had expected to observe increases in the carbonyl concentra-

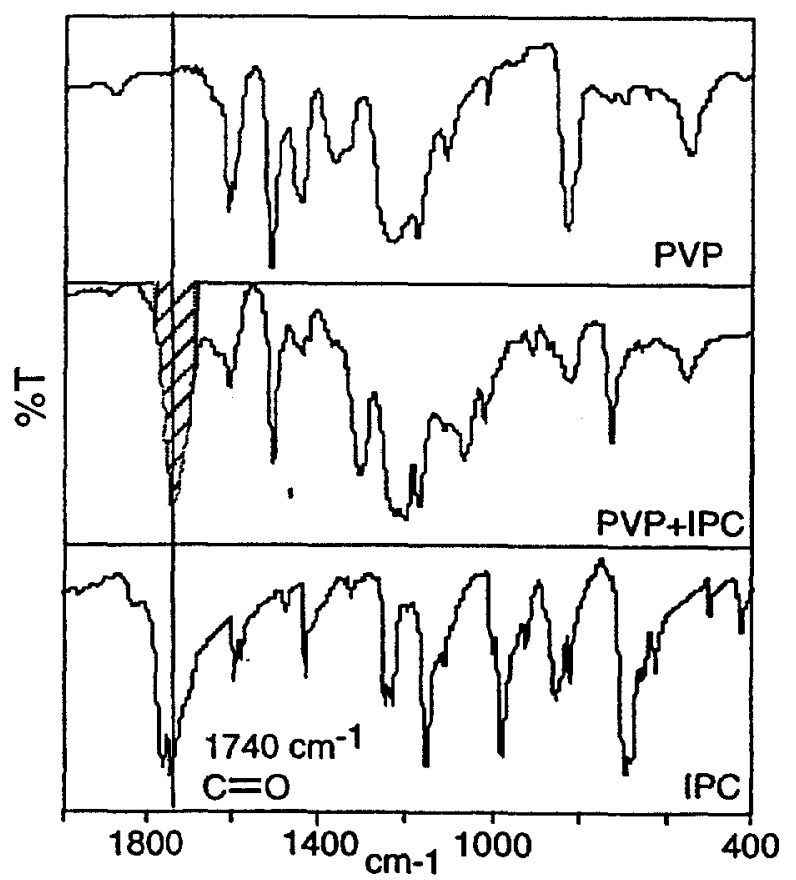

Fig. 6: IR spectroscopy used to measure the composition of the interfacial polymer. IR spectra of (a) PVP, (b) PVP and IPC, and (c) IPC. 
tions with reaction time due to an enhanced level of esterification of the phenolic group in the PVP chains. Instead, we found the measured IPC concentration / carbonyl concentration to be independent of time and to be constant for a particular solvent.

The wall thickness of interfacial polycondensation shells is, thus, controlled by the initial IPC concentration and the fraction reacted. The IR absorption was calibrated with phenyl benzoate (a material with similar bonding structure to the shells material that has a known carbonyl concentration). It is possible to relate the calibrated carbonyl absorption to the levels of IPC incorporated into the shells by using a mass balance. Figure 7 compares the measured final wall thicknesses of shells made in DEP and DCH solvents to the amount of IPC incorporated into the shell as calculated by mass balance. These data show that the amount of IPC incorporation is three times as high for the DEP shells as that for the DCH shell materials. We see graphically that use of poor solvents (e.g., DCH) result in thicker shell walls (than with DEP).

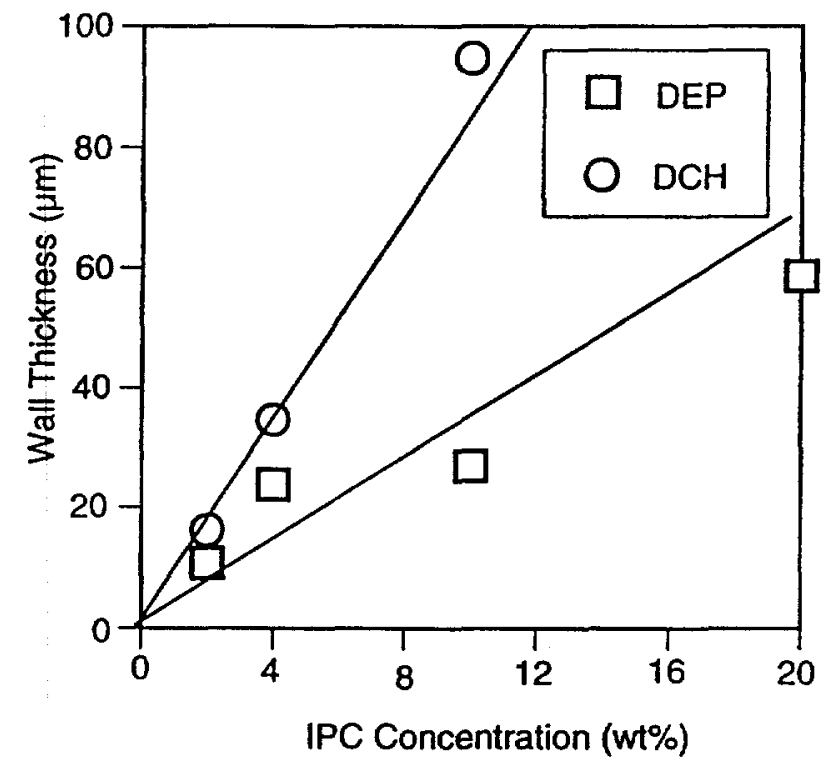

Fig. 7: Shell wall thickness vs. starting IPC wt\% for interfacial polycondensation shells using both DEP and DCH. The solid lines represent the values (i.e., slopes) one would obtain with a quantitative calibration with phenol benzoate and mass balance of IPC.

Thus, there are three important implications of the IR analysis regarding the growth of shells, the morphology, and the role of the oil phase solvent. First, shell walls grow thicker in DCH because less IPC is consumed per mass of shell. Only one third as much IPC is needed in DCH compared to DEP to produce a given thickness of shell. Second, as the PVP reacts with IPC at the interface a critical degree of reaction is reached at which point the polymer becomes insoluble and phase separates. Third, solubility controls the extent of reaction and the amount of IPC incorporation. The polymer is very soluble in DEP and fully reacts with the available IPC. The polymer is less soluble in DCH and precipitates when only one third as many sites are reacted. The compositional differences in the shell materials prepared in two different solvents also have been analyzed by a pyrolysis-GC-MS technique and nanoindentation hardness technique as discussed below. Both analyses confirm the magnitude of the composition differences in the shells.

\section{E. Pyrolysis-GC-MS}

By examining the total ion chromatogram (TIC) from the pyrolysis-GC-MS technique, it is possible to obtain information regarding the average bonding structure of a polymeric material. Our cross-linked materials can be thought of as a network copolymer because they are predominantly comprised of PVP repeat units (or PVP repeat units with singly reacted IPC side chains) and to a smaller fraction PVP repeat units that have reacted with the IPC at both ends and thereby cross-linked. Similarly, a PVP chain with IPC units that have reacted at only one end would appear exactly like a copolymer of vinyl phenol and IPC vinyl phenol. Analogous homopolymer and copolymers have been examined previously with the pyrolysisGC-MS technique. ${ }^{14-15}$ For a polymer that pyrolyzes via a beta-scission mechanism such as is known for polystyrene ${ }^{16}$ and its derivatives, ${ }^{17-18}$ we would expect the evolution and detection of not only monomeric species but also hybriddimer species (as well as trimers and tetramers to significantly attenuated levels at longer and longer retention times). Such behavior has been observed.

Because the major constituent of the shell material is PVP (or its copolymer), it is reasonable to compare pyrograms / TIC's of shell material to that of the unreacted PVP material. Similarities at short times (corresponding to small, more-volatile species related mostly to the PVP homopolymer) and the differences at long times (corresponding to larger, less-volatile species related mostly to the oligomeric hybrid species from the network copolymer) are obtained. The peaks at the short retention times reflect the "PVP-like" species given that they are present in both neat PVP and shell PVP. The peaks at the long retention times reflect the "cross-linked" nature of the shell. By comparing the relative peak areas, it is possible to get an indication of the amount of cross-linking occurring in the aggregate shell material. This argument is bolstered by the fact that similar behavior is observed with the pyrolysis of other linear copolymers. ${ }^{14-15}$

At the onset of the analysis, it was thought that if differences in the shell membrane existed as a function of shell wall thickness, it would be possible to detect by 
examining the shells generated for different reaction times. Two such shells were analyzed, one that had been reacted in DEP solvent for $10 \mathrm{~min}$. and the other that had been reacted for $90 \mathrm{~min}$. By comparing the relative peak areas of the homopolymer species to the network polymer species, it was evident that there were not any differences in the cross link densities with respect to time. This finding agrees with the IR analysis.

However, if the pyrograms of two shells generated with different organic solvents (DEP and DCH) are contrasted, we find striking differences. By comparing peaks at both long and short times, we find that the relative peak areas corresponding to shells produced with DEP have three times the network / hybrid species (due to the presence of cross-linked IPC) that shells produced with DCH have. The relative IPC content for the two types of shells is also in agreement with the $\mathbb{R}$ analysis. While we do not see observable changes in cross-link density with time (in one solvent system), we do see consistent differences for different organic solvents.

\section{F. Shell Hardness Measurement}

We were able to obtain differences in radial composition by probing the hardness of a shell wall fracture surface as a function of wall depth from the shell core layer to the exterior shell wall, thereby giving an indication of the evolution of the physical properties. This was accomplished by using a nanoindentation technique that has been developed using standard AFM instrumentation; ${ }^{19}$ it is capable of probing very local physical characteristics of materials by sampling areas in the size range of $50 \mathrm{~nm}^{2}$. The results obtained with this technique are provided in Figure 8. From these data it is apparent that there are significant differences in tensile moduli for both the changes in organic solvent (DEP and DCH) and hardness as a function of radial cross-section position. The shells tended to have higher moduli at the core of the membrane and lower moduli at the exterior of the membrane. The shells formulated in DEP also tended to be harder than the DCH equivalent.

It is also known that the mechanical moduli (both tensile and shear) of network polymers are inversely related to the molar mass between cross-links and directly related to the cross-link densities. ${ }^{20}$ Hence, it is reasonable to conclude that the shells generated in DEP have a greater cross-link density than the ones generated in DCH. The values from the microhardness measurements agree both in magnitude and direction with the IR measurements for cross-linking. The DEP shells are approximately three times as hard as the DCH shells.

\section{G. Permeation}

There are a number of reasons to include a discussion of permeation in a study of shell growth by interfacial polycondensation: a) permeation can be used as a means of characterizing dried shell materials, b) shell growth appears to be transport limited, c) NIF shells will ultimately be diffusion filled with gaseous fuel.

1. Solvent permeation. Permeation, the product of both diffusivity $(D)$ and solubility $(S)$, can be measured directly with a mass uptake / sorption experiment in which the mass of the sample is subjected to saturated vapor of various permeants such as tetrahydrofuran (THF) and ethanol (EtOH); the resulting mass changes are measured accurately with a Cahn microbalance over extended periods. The experiments can be run in either adsorption (increasing mass from a neat material) or desorption mode (decreasing mass from a saturated state). For Fickian diffusion the following approximation applies at short experimental times: ${ }^{21}$

$$
\frac{M_{t}-M_{0}}{M_{-}-M_{0}}=\frac{4}{\pi L} \sqrt{D t} \text { for adsorption }
$$

$$
\frac{M_{-}-M_{t}}{M_{-}-M_{0}}=\frac{4}{\pi L} \sqrt{D t} \text { for desorption }
$$

where $M_{o}$ is the original mass of the dry membrane material, $M_{t}$ is the mass of the material at any time, $t, M_{\infty}$ is the mass at infinite time, i.e., at saturation, $L$ is the

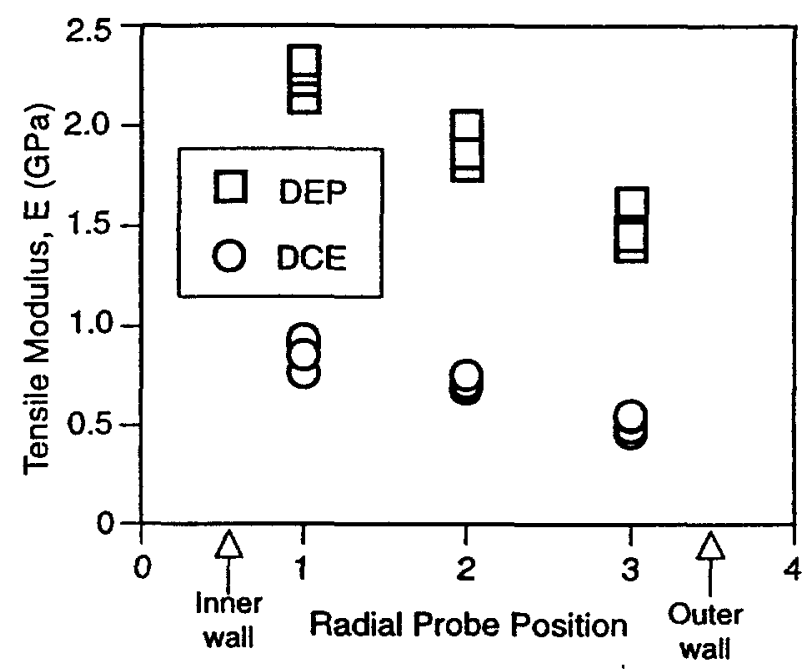

Fig. 8: Comparison of tensile moduli for shells formed in both DEP and DCH. The tensile moduli are measured for three positions in the shell; 1) immediately inside the core layer (not including the smooth core layer shown in Fig. 4), 2) half way between the inside and outside surfaces of the shell, and 3) immediately on the inside of the exterior surface so as to avoid edge effects. 
characteristic diffusional length. For fragmented shell materials with diffusant vapor exposure on both side, $L$ is half of the actual wall thickness.

The typical information generated in a mass uptake / sorption experiment is shown in Figure 9 for both the adsorption mode and the complement of desorption, i.e., 1 $\left(M_{\infty}-M_{t}\right) /\left(M_{\infty}-M_{o}\right)$. It should be noted that both curves displayed in Figure 9 are good examples of Fickian diffusion, i.e., a linear region representing the data for the first $50-60 \%$ of the sorption curve. Deviations from Fickian behavior frequently occurs when the relaxation rate of the polymer chains is on the same order of magnitude as the diffusion rate (case II diffusion). ${ }^{2-23}$ The adsorption and desorption curves do not overlap because $D$ is not constant throughout the experiment; ${ }^{21.24} D$ is expected to change given the relatively high solubility of the diffusants in the polymer. The differences in sorption behavior at longer times can be readily explained by plasticizing effects of both THF and EtOH. The oscillations observed in the absorption data at steady state in Figure 9 are believed simply to be due to ambient temperature fluctuations (and consequently oscillations in the equilibrium permeant vapor pressure; vapor pressure values are strongly temperature dependent in the neighborhood of the boiling point). A number of adsorption and desorption measurements were obtained for shells generated from both DCH and DEP solvents (Table 1). From these data, it is apparent that both diffusants, THF and EtOH, permeate the shell material generated with DCH faster than the shell made with DEP (approximately 5-10 times faster). It is also evident that THF travels quicker through and to a greater extent than the EtOH diffusant.

The mass uptake data can be compared to other techniques measuring permeability such as $x$-ray fluorescence (XRF). In the XRF experiment, shells are diffusionfilled with argon $(\mathrm{Ar})$ and transferred to an $\mathrm{x}$-ray fluorom-

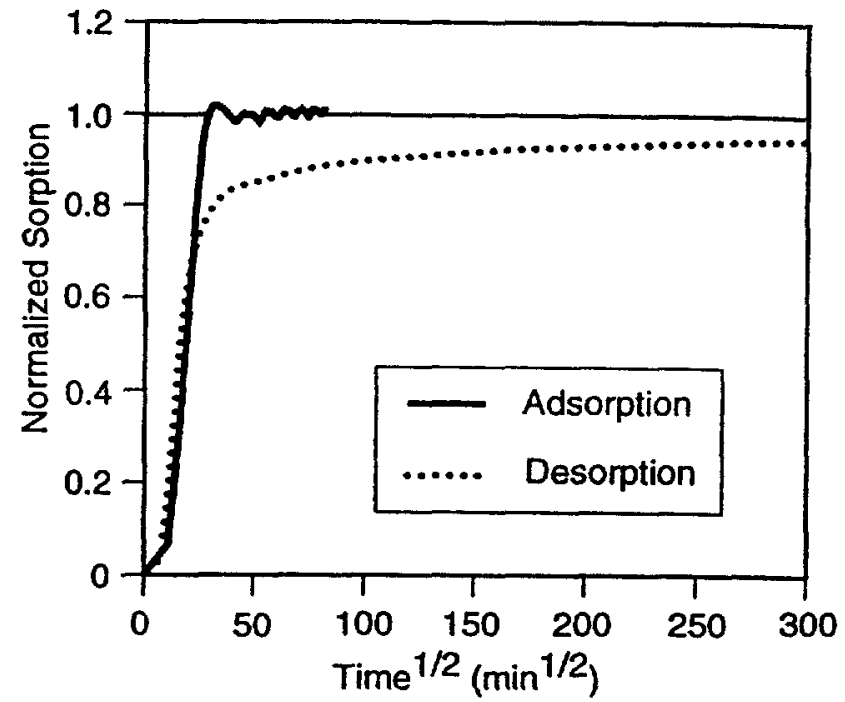

Fig. 9: Typical data obtained from a sorption experiment. Each set of data is normalized with respect to both the initial sample weight and the final sample weight. Both the adsorption data (solid line) and the complement of the desorption data (dottedline) are shown for convenience.

eter. The Ar permeation through the shell is measured as the decay in the fluorescence signal (which is proportion to the Ar concentration contained within the shell). Permeability of argon gas through DEP shells is $10 \pm 5 \mathrm{sccm}$ psi/ $\mathrm{cm} \mathrm{cm}^{2} \mathrm{~m}$ while the permeability of Ar through DCH shells is $80 \pm 40 \mathrm{sccm}$ psi $/ \mathrm{cm} \mathrm{cm}^{2} \mathrm{~m}$. From these data, a ratio of permeabilities for the same permeant was approximately 8 (as opposed to 14 for THF and 5 for EtOH).

2. Relationship of shell growth kinetics to IPC permeation. Further examination of diffusion processes with respect to interfacially polymerized shells is warranted given that the polymerization process (after the initial thin

Table 1 Solvent Permeation Data for Interfacial Polycondensation Polymers

\begin{tabular}{|c|c|c|c|c|c|c|c|c|}
\hline $\begin{array}{l}\text { Oil Phase } \\
\text { Solvent }\end{array}$ & $\begin{array}{l}\text { Wt\% } \\
\text { IPC }\end{array}$ & $\begin{array}{l}\text { Wall } \\
(\mu \mathrm{m})\end{array}$ & $\begin{array}{l}\text { Per- } \\
\text { meant }\end{array}$ & $\begin{array}{l}D_{\text {sss }_{2}} \\
\left(\mathrm{~cm}^{\prime} / \mathrm{s}\right)\end{array}$ & $\begin{array}{l}D_{\operatorname{des}_{2}} \\
(\mathrm{~cm} / \mathrm{s})\end{array}$ & $S^{\dagger}$ & $\begin{array}{l}\mathrm{P}_{\mathrm{xds}_{2}} \\
\left(\mathrm{~cm}^{2} / \mathrm{s}\right)\end{array}$ & $\begin{array}{l}\left.P_{\operatorname{des}^{2}}{ }^{2} / \mathrm{s}\right) \\
\left(\mathrm{cm}^{2} / \mathrm{s}\right.\end{array}$ \\
\hline \multirow[t]{2}{*}{$\mathrm{DCH}^{*}$} & 10 & 94 & EtOH & $4.7 \times 10^{-9}$ & $3.7 \times 10^{-8}$ & 0.25 & $1.2 \times 10^{-9}$ & $9.2 \times 10^{-9}$ \\
\hline & & & & $1.5 \times 10^{-8}$ & $2.1 \times 10^{-8}$ & 0.27 & $3.7 \times 10^{-9}$ & $5.3 \times 10^{-9}$ \\
\hline $\mathrm{DCH}$ & 10 & 94 & THF & $4.0 \times 10^{-8}$ & $8.0 \times 10^{-8}$ & 0.83 & $3.3 \times 10^{-8}$ & $6.6 \times 10^{-8}$ \\
\hline $\mathrm{DCH}$ & 10 & 94 & EtOH & $1.1 \times 10^{-8}$ & $3.9 \times 10^{-8}$ & 0.24 & $2.7 \times 10^{-9}$ & $9.2 \times 10^{-9}$ \\
\hline \multirow[t]{2}{*}{ DEP* } & 4 & 10 & EtOH & $7.6 \times 10^{-10}$ & $3.9 \times 10^{-10}$ & 0.46 & $3.5 \times 10^{-10}$ & $1.8 \times 10^{-10}$ \\
\hline & & & & $1.1 \times 10^{-9}$ & $3.6 \times 10^{-10}$ & 0.42 & $4.7 \times 10^{-10}$ & $1.5 \times 10^{-10}$ \\
\hline DEP & 4 & 10 & THF & $2.7 \times 10^{-9}$ & $7.3 \times 10^{-10}$ & 0.89 & $2.4 \times 10^{-9}$ & $6.5 \times 10^{-10}$ \\
\hline
\end{tabular}

'Solubility is defined as the ratio of permeant uptake $(\Delta M$ at $t=\infty)$ to initial mass of polymeric membrane material $\left(M_{0}\right)$. ${ }^{\ddagger}$ Permeation values calculated from desorption data are calculated using the solubility as measured in the adsorption experiment. "Duplicated entries in the table indicate a repeated experiments at nominally the same conditions. 
film is formed at the interior of the shell) is thereafter limited by diffusion of the limiting reagent, IPC, and this relates to other measures of permeation. It is believed (and experiment evidence suggest) that the true reaction proceeds according to the following expression:

$$
\frac{\partial W}{\partial t} \propto \frac{\partial[I P C]}{\partial t}=k[I P C]\left[P V P^{*}\right]+\frac{\partial}{\partial x}\left(D \frac{\partial[I P C]}{\partial x}\right)
$$

where $W$ is the wall thickness of the shell, $k$ (liter mol-1 $^{-1}$ $\left.\sec ^{-1}\right)$ is the rate constant of presumably a second order reaction, $[I P C]$ is the molar concentration of isopthaloyl dichloride, $\left[P V P^{*}\right]$ is the molar concentration of deprotonated vinyl phenol groups, $D$ is the diffusion constant (of IPC) in the forming membrane, $t$ is reaction time, and $x$ is the shell wall position. The first term represents the reaction kinetics and the second term represents the diffusional flux of IPC according to Fick's second law. It is presumed that the IPC reacts rapidly once it reaches the surface of the growing membrane. If initial boundary conditions at both surfaces could be maintained, steady state conditions eventually would be obtained resulting in a linear IPC concentration gradient through the shell; however, because we have essentially a number of small batch reactors during shell growth, we observe transient conditions resulting in an error function distribution of IPC concentration with respect to shell wall radial position.

It is possible to apply the same principle, used to examine the permeation of THF and EtOH through shell membranes, to examine the mechanism of shell growth. Instead of observing the sorption of an "inert" solvent, we can observe the transport of the key reactants. Because we have postulated that the observed interfacial reaction is limited by diffusion, we have utilized the same Fickian analysis, described above for the motion of the small molecules (THF and EtOH) through the membrane material, to characterize IPC motion through the forming membrane. It is presumed that the reaction predominantly occurs at the outermost surface of the forming membrane, i.e., at the interface of IPC / solvent oil phase and PVPloaded aqueous phase. Thus, an IPC molecule must diffuse from the core fluid interface through the forming membrane to the exterior surface when it is met by a deprotonated repeat unit of the PVP chain.

If the wall thickness data for various IPC concentrations in a singular solvent (such as data from Figure 3 ) are normalized by a characteristic length scale, $L$, (which is taken to be the final wall thickness of the shell) and plotted on a $L$-normalized time scale as suggested by Fickian diffusion and Eq. 1, a similar type of behavior is observed
(Figure 10). With this rescaling, we find reasonable overlap of the data into one coherent trend (similar to solvent sorption data). This corroborates the assertion that this interfacial reaction scheme is in fact limited by the diffusion of IPC. Furthermore, these data can be fitted to a model incorporating both aspects of the diffusion and reaction kinetics, and a best fit to their expression is provided as the solid curve in Figure 10. See Takagi, et al.," for the details of the Osaka University model.

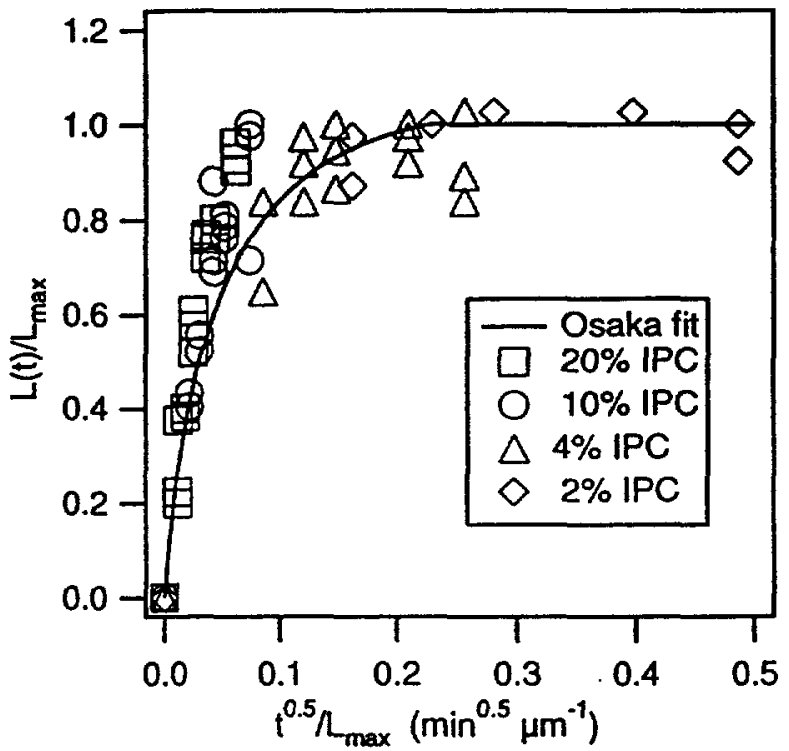

Fig. 10: Rescaled shell wall thickness data for various wt\% IPC in DCH solvent. The characteristic wall thickness, $L$, in the expression for Fickian diffusion (Eq 1) is taken to be $L_{\max }$ for each IPC concentration. The solid line represents a best fit to the Osaka University model. ${ }^{11}$

With this protocol, we can calculate the diffusion coefficient for IPC in two shell materials examined in the solvent sorption experiments. Calculated diffusion coefficient for IPC through the forming shell using either DEP or DCH as solvents are $2.30 \times 10^{-8} \mathrm{~cm}^{2} / \mathrm{sec}$ and $2.82 \times 10^{-7} \mathrm{~cm}^{2} / \mathrm{sec}$, respectively. Both values are on the correct order of magnitude one would expect for small molecule motion through a rubbery polymer. ${ }^{21}$ When adjusted for solubility, these values give a ratio for the permeation of IPC through the two types of membranes, $\mathrm{P}_{\mathrm{DCH}} / \mathrm{P}_{\mathrm{DEP}}$, equal to 12 . This value agrees (in both direction and magnitude) with both the sorption measurements and the XRF measurements for the two materials.

From the agreement for each of the techniques (for diffusion measurements like this, agreement within an order of magnitude is generally considered good), it is possible to conclude that the results are real. The two shell materials 
have distinct transport and shell growth properties; due to the particular shell growth mechanism, these properties are interrelated. It is apparent that the diffusant traverses the materials fabricated in DCH about ten-fold times as fast as the materials generated from DEP no matter which diffusant is chosen (Ar, ethanol, THF, or IPC). This undoubtedly is due to differences in cross-linking.

\section{SUMMARY}

Major differences in the interfacial polycondensation shell properties were found as a function of organic phase solvent (DEP and DCH). These differences were manifested in terms of the kinetics of shell deposition, ultimate wall thickness (for a given IPC starting concentration) and surface finish. The shells characteristics made with DCH tended to grow much quicker, incorporate less IPC (IR, Pyrolysis-GC-MS), cross-link to a lower extent (microhardness), maintain an inferior surface (AFM patch scans), and exhibit enhanced transport characteristics (THF, $\mathrm{EtOH}, \mathrm{Ar}$, and IPC permeation measurements) relative to the DEP equivalent.

These observations are internally consistent, and they are most likely due to the specifics of the reaction in the interfacial region. Shell deposition occurred via a diffusion limited reaction, and can be examined with standard Fickian analyses; these data can be fitted equally as well to the Osaka University model." The crossover from a reaction limited mechanism to a diffusion limited mechanism is evidenced by the change in shell wall morphology at the innermost surface in comparison to the bulk of the shell wall. It is believed that DEP is a better solvent for the reacting PVP polyelectrolyte, and the polymer chains tend to remain solvated longer than the DCH counterpart; this additional time enables a larger fraction of the IPC to react at both ends, thereby accounting for the differences in shell properties, i.e., hardness, deposition rate, and surface roughness.

\section{ACKNOWLEDGMENTS}

This work was performed under the auspices of the U. S. Department of Energy by the Lawrence Livermore National Laboratory under Contract No. W-7405-ENG-48. Special thanks are extended to Medhi Balooch (microhardness), Ed Lindsey (SEM), and Craig Moore (XRF) for their individual assistance in the collection of experimental data from the respective techniques.

\section{REFERENCES}

1. S. W. Haan., et al., "Design and Modeling of Ignition Targets for the National Ignition Facility," Phys. Plasmas 2, 2480 (1995).

2. R. Cook, "Production of Hollow Microspheres for Inertial Confinement Fusion Experiments," in Hollow and Solid Spheres and Microspheres-Science and Technology Associated with Their Fabrication and Application, D. L. Wilcox et al., Eds. (Materials Research Society, Pittsburgh, PA), pp. 101-112 (1995).

3. S. A. Letts, et al., "Preparation of Hollow Shell ICF Targets Using a Depolymerizing Mandrel," in Hollow and Solid Spheres and Microspheres-Science and Technology Associated with Their Fabrication and Application, D. L. Wilcox et al., Eds. (Materials Research Society, Pittsburgh, PA), pp. 125-130 (1995).

4. S. A. Letts, et al., "Fabrication of Special Inertial Confinement Fusion Targets Using a Depolymerizable Mandrel Technique," J. Vac. Sci. Technol. A 14, 1015 (1996).

5. S. A. Letts, et al., "Fabrication of Polymer Shells Using a Depolymerizable Mandrel," Fusion Technol., 28, 1797 (1995).

6. M. Takagi, T. Norimatsu, T. Yamanaka, and S. Nakai, "Development of Deuterated Polystyrene Shells for Laser Fusion by Means of a Density-matched Emulsion Method," J. Vac. Sci. Technol. A, 9(4), 2145 (1991).

7. G. Wilemski, et al., "Prediction of Phase Separation during the Drying of Polymer Shells," Fusion Technol., 28, 1773 (1995).

8. T. Boone, et al., "Modeling of Microencapsulated Polymer Shell Solidification," in Hollow and Solid Spheres and Microspheres-Science and Technology Associated with Their Fabrication and Application, D. L. Wilcox et al., Eds. (Materials Research Society, Pittsburgh, PA), pp. 193-198 (1995).

9. Y. A. Merkuliev, et al., "Study of Production and Quality of Large (1-2 mm) Polystyrene Hollow Microspheres," in Hollow and Solid Spheres and Microspheres-Science and Technology Associated with Their Fabrication and Application, D. L. Wilcox et al., Eds. (Materials Research Society, Pittsburgh, PA), pp. 119-124 (1995).

10. A.I. Nikitenko, S.M. Tolkonnikov, and R. Cook, "The Design of the Ballistic Furnace and Initial Microshell Formation," Fusion Technol., (1997), this issue.

11. M. Takagi, et al., "Development of Foam Shell with Plastic Ablator for Cryogenic Laser Fusion Target," $J$. Vac. Sci. Technol. A, 11(5), 2837 (1993).

12. R. Arshady, "Preparation in Microspheres and Microcapsules by Interfacial Polycondensation Techniques, “J. Microencapsulation, 6(1), 13, (1989).

13. B. Rempp and E.W. Merrill, Polymer Synthesis, 2nd ed., New York: Huthig \& Wepf, (1991). 
14. S. Mao, H. Ohtani, and S. Tsuge, "Compositional Analysis of Multicomponent Acrylic Resins by Pryolysis - Capillary Gas Chromatography", J. Anal. Appl. Pyrol., 33, 181, (1995).

15. T. Isemura, Y. Jitsugiri, and S. Yonemori, "Compositional and Sequential Analysis of Poly(ethylenetetraethylene) by Pyrolysis-Gas Chromatography", $J$. Anal. Appl. Pyrol. 103, 109. (1995).

16. J.E. Mark, (ed), Physical Properties of Polymers, New York: AIP Press, (1996).

17. F. Bertini, G. Audisio, and J. Kiji, "Thermal Behavior and Degradation Mechnamism of Brominated Polystyrenes," J. Anal. Appl. Pyrol., 33, 213, (1995).

18. F. Bertini, G. Audisio, and J. Kiji, "Thermal Degradation of Chlorinated Polystyrenes," J. Anal. Appl. Pyrol., 28, 205, (1994).

19. M. Balooch, A.J. Lundkvist, J. Kinney, S.J. Marshall, and W.J. Siekhaus, "Viscoelastic Properties of Decalcified Human Dentine Measured in Water by an AFMBased Indentation Technique," to be published.

20. J.M.G. Cowie, Polymers: Chemistry \& Physics of Modern Materials, 2nd ed., London: Blackie \& Son, Ltd., (1991).

21. J. Crank, and G.S. Park, Diffusion in Polymers, New York: Academic Press, Inc., (1968).

22. D.J. Ensore, H.B. Hoppenberg, V.T. Stannett, "Effect of Particle Size on the Mechanism Controlling n-hexane Sorption in Glassy Polystyrene," Polymer, 18, 793. (1977).

23. A.R. Berens, H.B. Hoppenberg, "Diffusion and Relaxation in Glassy Polymer Powders: 2. Separation of Diffusion and Relaxation Parameters," Polymer, 19, 489, (1978).

24. K.E. Miller, R.H. Krueger, and J.M. Torkelson, "Mobility-Sensitive Fluorescence Probes for Quantative Monitoring of Water Sorption and Diffusion in Polymer Coatings," Journal of Polymer Science:

Part B: Polymer Physics, 33, 2343 (1995). 


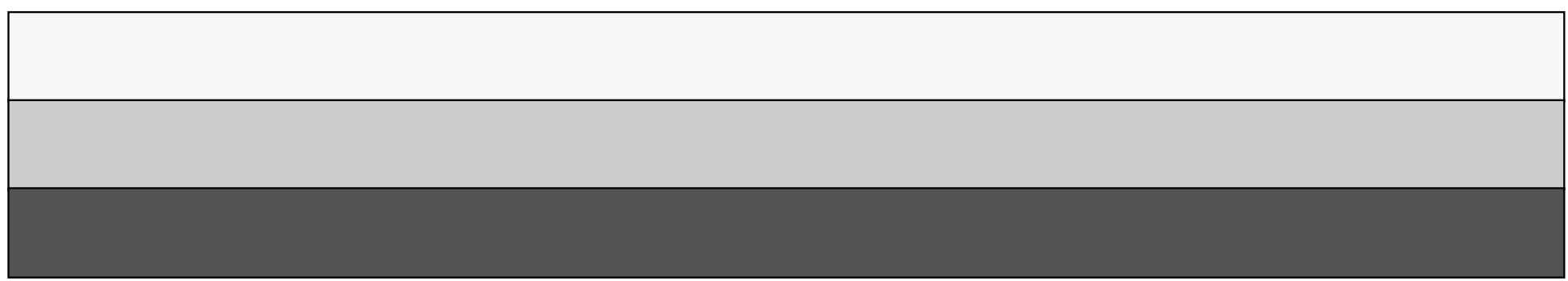

